\title{
Antimicrobial mechanism of epigallocatechin gallate and gallocatechin gallate: They target 1-deoxy-D-xylulose 5-phosphate reductoisomerase, the key enzyme of the MEP terpenoid biosynthetic pathway
}

Xian Hui, Shui-Hong Hua, Qian-Qian Wu, Heng Li and Wen-Yun Gao*

National Engineering Research Center for Miniaturised Detection Systems and College of Life Sciences, Northwest University, 229 North Taibai Road, Xi'an, Shaanxi 710069, China

*Prof. Dr. Wen-Yun Gao: To whom correspondence should be addressed (Fax: +86 29 88303572;

Tel: +86 2988303446 ext. 832; E-mail: gaowenyun@nwu.edu.cn) 


\begin{abstract}
The catechins EGCG and GCG show a variety of pharmacological activities, especially an antibacterial capacity, but their modes of antimicrobial action have not been fully elucidated. 1-Deoxy-D-xylulose 5-phosphate reductoisomerase (DXR), the first key enzyme in the MEP pathway for terpenoid biosynthesis, is a recently validated antimicrobial target. In order to disclose the antibacterial mechanism of EGCG and GCG, the DXR inhibitory activity of them was investigated in this study. The data show that EGCG and GCG both could specifically suppress the activity of DXR, with EGCG exhibiting relatively low effect against DXR $\left(\mathrm{IC}_{50}\right.$ about $210 \mu \mathrm{M})$ and GCG displaying strong activity $\left(\mathrm{IC}_{50} 27.5 \mu \mathrm{M}\right)$. In addition, studies on inhibition kinetics of the catechins against DXR demonstrate that they are competitive inhibitors of DXR against DXP and uncompetitive inhibitors with respect to NADPH. Meanwhile, the possible interactions between DXR and the catechins were simulated via docking experiments.
\end{abstract}

\title{
Keywords:
}

EGCG; GCG; Mode of antimicrobial action; 1-Deoxy-D-xylulose 5-phosphate reductoisomerase; MEP pathway; Specific inhibition.

\section{Chemical compounds studied in this article}

Epigallocatechin gallate (PubChem CID: 65064); Gallocatechin gallate (PubChem CID: 199472).

\section{Abbreviations}

CIAP Calf intestine alkaline phosphatase 


\author{
DLS Dynamic light scattering \\ DNPH 2,4-Dinitrophenylhydrazine \\ DXP 1-Deoxy-D-xylulose 5-phosphate \\ DXR 1-Deoxy-D-xylulose 5-phosphate reductoisomerase \\ EGCG Epigallocatechin gallate \\ FabG $\quad \beta$-Ketoacyl-acyl carrier protein reductase \\ GCG Gallocatechin gallate \\ MEP 2-Methyl-D-erythritol 4-phosphate \\ VC Vitamin C (ascorbic acid).
}

\title{
1. Introduction
}

EGCG (1, Fig. 1) is one of the major catechin compounds found in unfermented green tea, partially fermented oolong tea, and fully fermented black tea [1]. Among all the tea catechins, EGCG has received the most attention and research has shown that it exhibits a broad range of physiological effects such as anti-diabetes, anticancer, antivirus, and antibacterial activity [2-4] with its antibacterial property being specially emphasized. The investigation on the antibacterial mechanism of the compound has indicated that: 1) EGCG can directly bind with peptidoglycan, damaging the cell wall and interfering with its biosynthesis [4]; 2) EGCG can react with dissolved oxygen in aqueous solution, generating hydrogen peroxide that possesses bactericidal effects [5]; 3) EGCG can inhibit extracellular release of Vero toxins from enterohemorrhagic $E$. coli [6]; 4) EGCG inhibits the bacterial type II fatty-acid synthase [7]. GCG (2, Fig. 1), the C-2 
epimer of $\mathbf{1}$, is another major catechin compound isolated from tea. GCG has shown better inhibitory activity against Bacillus cereusis than EGCG and also shown suppressive effect against the release of Vero toxins [6], but these have not drawn much attention.

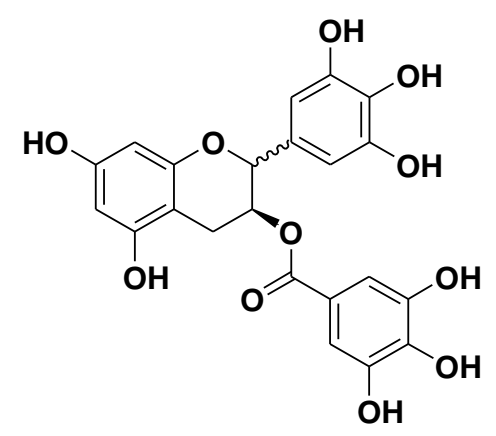

\section{1: EGCG, 2R; 2: GCG, 2S}

Fig. 1 Structures of EGCG and GCG.

Recently, a novel terpenoid biosynthetic pathway, namely the MEP pathway has been established. Research has shown that this pathway is only operative in microorganisms including human pathogens, but not in human beings. Furthermore, it is also essential for the survival of the microbes because disruption of the pathway is lethal. Thus the MEP pathway has been regarded as a useful target for screening of antimicrobial agents $[9,10]$. DXR is one of the key enzymes of the pathway, catalyzing the rate-limiting step, namely the reductive isomerization of DXP (3, Fig. 2) to MEP (4) in the presence of NADPH and a divalent metal ion. It has drawn so much attention and lots of research has been centered on it, leading to the elucidation of fosmidomycin (5) and FR900098 (6), two natural phosphonates which not only potently inhibit DXR, but have good activity against pathogenic microorganisms as well [11]. Further clinical data have pointed out that $\mathbf{5}$ used alone or in combination with clindamycin are somewhat effective in treating malaria 
caused by Plasmodium falciparum [12]. Plants produce incalculable natural metabolites, many of which display antimicrobial activity. Therefore, it might be a credible source for seeking DXR inhibitors. But up to date, only Kaiser and co-workers tried to look for DXR inhibitors from Mediterranean plants and found that the leaf extracts of Cercis siliquastrum exhibit strong inhibitory activity against DXR. Unfortunately, no specific compound has been elucidated [13].

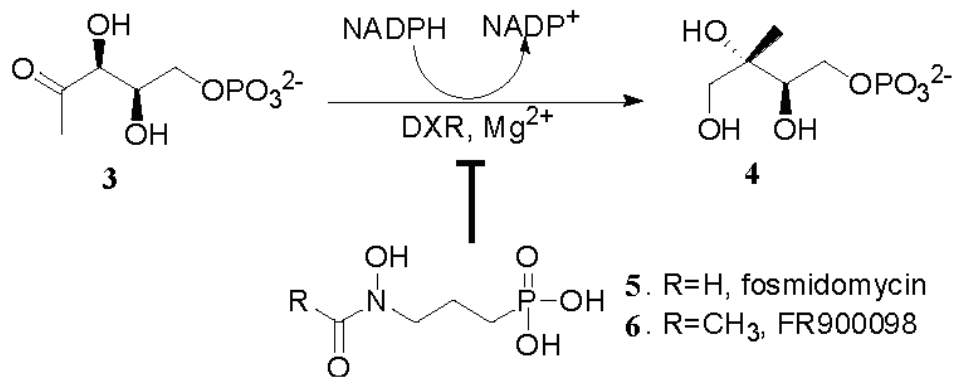

Fig. 2 The rate-limiting step of the MEP pathway mediated by DXR and its inhibitors.

In our previous study, we found that tea extracts exhibit moderate DXR inhibitory activity and the major active components seem to be polyphenols, especially EGCG and GCG [14]. We therefore further determined the details of DXR inhibition of the four theaflavin compounds [15] and the catechins EGCG and GCG so as to deepen our understanding of the antibacterial mechanism of these compounds and broaden our knowledge of tea. Furthermore, we could find through the study the DXR inhibitors of plant origin which possess completely different structural features compared to fosmidomycin. In this paper, we would disclose all the details about the DXR inhibitory activity of EGCG and GCG. 


\section{Materials and methods}

\subsection{Materials}

EGCG and GCG were purchased from Chengdu Biopurify Phytochemicals Ltd. (Chengdu, China) and the stock solutions of them $(10 \mathrm{mg} / \mathrm{mL})$ were prepared in $40 \%$ aqueous DMSO or $0.1 \%$ Triton X-100 just before use. NADPH was purchased from Gen-View Scientific Inc. (Tallahassee, USA); Fosmidomycin was from Toronto Research Chemicals Inc. (North York, Canada); CIAP was purchased from Takara (Dalian, China); Triton X-100 and DNPH were purchased from Sinopharm Chemical Reagent Co., Ltd. (Beijing, China). DXP was synthesised according to the procedure previously reported [16]. Preparation of E. coli DXR was carried out in accordance with the published method [17]. HPLC grade methanol and acetonitrile were purchased from Sigma-Aldrich Chemical Co. (Shanghai, China). All other chemicals involved in this study are of analytical grade.

\subsection{Stability of EGCG and GCG under DXR assay conditions}

Certain amount of EGCG and GCG was diluted separately into $50 \mathrm{mM}$ Tris-HCl buffer (pH 7.0) to a final concentration of $50 \mu \mathrm{M}$ in the absence and presence of $1 \mathrm{mM} \mathrm{VC}$. The solutions were then incubated at $37^{\circ} \mathrm{C}$ and determined by HPLC every half an hour on an Agilent 1200 HPLC system equipped with a DAD detector. The HPLC conditions: Column, Shim-pack VP-ODS column $(250 \times 4.6 \mathrm{~mm}, 4.6 \mu \mathrm{m})$; Mobile phase: methanol-water (containing $1 \%$ acetic acid, v/v); Gradient: 0 min, 15\% methanol; 12-13 min, 55\% methanol; 20-25 min, 15\% methanol. Detection 
wavelength: $280 \mathrm{~nm}$; Flow rate: $0.8 \mathrm{~mL} / \mathrm{min}$; Injection volume, $20 \mu \mathrm{L}$; Column temperature:

ambient.

\subsection{Determination of inhibitory activity of EGCG/GCG against E. coli DXR}

The inhibitory activity of EGCG/GCG against $E$. coli DXR was determined by using the pre-column derivatization HPLC method published by this group with minor modification [15]. Assay mixture consists of $50 \mathrm{mM}$ Tris- $\mathrm{HCl}$ ( $\mathrm{pH} 7.0), 5 \mathrm{mM} \mathrm{MgCl}, 0.5 \mathrm{mM}$ NADPH, $1 \mathrm{mM} \mathrm{VC}$, $2 \%$ (W/V) DMSO or $0.01 \%$ Triton X-100, and $4 \mu \mathrm{g} / \mathrm{mL}$ DXR in a final volume of $100 \mu \mathrm{L}$.

EGCG or GCG was added to the mixture respectively before the addition of DXP (end concentration $1 \mathrm{mM}$ ) to start the reaction. In a control assay, fosmidomycin (5) was used instead of the catechins. The reaction mixture was incubated at $37^{\circ} \mathrm{C}$ for $30 \mathrm{~min}$ before DXR was inactivated by boiling for 3 minutes. Subsequently, $10 \mu \mathrm{L}$ CIAP buffer ( $1 \mathrm{M}$ Tris-HCl, $1 \mathrm{M} \mathrm{NaCl} 2$ $50 \mathrm{mM} \mathrm{MgCl} 2, \mathrm{pH} 9.5$ ) and 2 units of CIAP were added and the mixture was incubated at $37^{\circ} \mathrm{C}$ for $120 \mathrm{~min} .10 \mu \mathrm{L}$ DNPH solution (120 mM in 30\% perchloric acid, V/V) and $280 \mu \mathrm{L}$ methanol were supplemented. After incubated at $37{ }^{\circ} \mathrm{C}$ for another $45 \mathrm{~min}$, the mixture was centrifugated and the supernatant was used for HPLC analysis. HPLC conditions: Column, Shim-pack VP-ODS column $(250 \times 4.6 \mathrm{~mm}, 5 \mu \mathrm{m})$. Injection volume: $60 \mu \mathrm{L}$. acetonitrile-water gradient: $0 \mathrm{~min}, 40 \%$ acetonitrile; $17 \mathrm{~min}, 80 \%$ acetonitrile; $18-20 \mathrm{~min}, 40 \%$ acetonitrile. Detection wavelength: 360 nm. Flow-rate: $0.7 \mathrm{~mL} / \mathrm{min}$. Column temperature: $30{ }^{\circ} \mathrm{C}$.

\subsection{Measurement of CIAP inhibitory effect of EGCG/GCG and other compounds}


The HPLC method mentioned above was also utilized to measure the inhibitory effects of

EGCG/GCG and other compounds such as VC, Triton X-100, and baicalein against CIAP. The reaction mixture comprises of $10 \mu \mathrm{L}$ CIAP buffer and 2 units of CIAP in an end volume of 100 $\mu \mathrm{L}$. Then $1 \mathrm{mM}$ VC, or $0.1 \%$ Triton X-100, or $0.5 \mathrm{mM}$ baicalein, or EGCG/GCG (for each compound, two concentrations, namely $50 \mu \mathrm{M}$ and $500 \mu \mathrm{M}$ were applied) plus $1 \mathrm{mM}$ VC were added to the mixtures respectively before the addition of DXP (final conc. $1 \mathrm{mM}$ ) to initiate the reaction. In a control assay, only $1 \mathrm{mM}$ DXP was supplemented. The reaction mixtures were incubated at $37^{\circ} \mathrm{C}$ for $120 \mathrm{~min}$ and CIAP was inactivated in boiling water for $3 \mathrm{~min}$ before they were derivatised with DNPH and determined with the HPLC method.

\subsection{Analysis of the particle size by DLS}

The particle size of EGCG/GCG in Tris-HCl buffter $(50 \mathrm{mM}, \mathrm{pH} 7.0)$ containing $1 \mathrm{mM} \mathrm{VC}$ and $2.0 \% \mathrm{DMSO}(\mathrm{V} / \mathrm{V})$ was analyzed at room temperature in the absence and presence of DXR (end concentration: $4 \mu \mathrm{g} / \mathrm{mL}$ ) on a DLS analyzer (NICOMP-380, Particle Sizing Systems Inc., Santa Barbara, Calif., USA). The detector time was set to $10 \mathrm{~min}$. The concentration of EGCG or GCG was at 2-3 times of its $\mathrm{IC}_{50}$ value. A solution of $2.0 \%$ aqueous DMSO plus $1 \mathrm{mM} \mathrm{VC}$ was used as a reference.

\subsection{Interaction between EGCG/GCG and DXR measured by UV spectrometry}

The UV absorbance at $400 \mathrm{~nm}\left(\mathrm{~A}_{400}\right)$ of the solutions containing EGCG/GCG and DXR was

monitored by using the method published by Li et al [18]. Details of the method were as follows: 
To phosphate buffer solutions (2.5 mM, pH 7.0) different additives such as EGCG, GCG, DXR, and VC were added respectively. The compositions of each solution were listed in Table 1 . Then each of the solution was kept at room temperature and the $\mathrm{A}_{400}$ was monitored at every 30 seconds by an Agilent 8453 UV spectrophotometer. Meanwhile, the solutions were also monitored by the DLS analyzer every 30 min to analyze if there was any particles formed.

Table 1 The compositions of each solution (in $2.5 \mathrm{mM}$ phosphate buffer, $\mathrm{pH}$ 7.0) prepared to determine the interaction between EGCG/GCG and DXR

\begin{tabular}{cccccccccccc}
\hline & soln. 1 & 2 & 3 & 4 & 5 & 6 & 7 & 8 & 9 & 10 \\
\hline $\operatorname{EGCG}(200 \mu \mathrm{M})$ & + & - & + & - & + & - & + & - & - & - \\
$\operatorname{GCG}(200 \mu \mathrm{M})$ & - & + & - & + & - & + & - & + & - & - \\
$\operatorname{DXR}(3 \mu \mathrm{M})$ & + & + & - & - & + & + & - & - & + & + \\
$\operatorname{VC}(1 \mathrm{mM})$ & - & - & - & - & + & + & + & + & & + & + \\
\hline
\end{tabular}

\subsection{Docking experiment}

The docking experiments were carried out employing Autodock4.2.6 and the results were shown by Chimera1.10.1. The profiles of the crystal of DXR-NADPH- $\mathrm{Mg}^{2+}$-fosmidomycin quaternary complex were extracted from Protein Data Bank (PDB accession code 2EGH). Fosmidomycin was removed and then the docking simulation was performed using the EGCG or GCG as a ligand and the mimic DXR-NADPH- $\mathrm{Mg}^{2+}$ ternary complex as a receptor. The conformation with the lowest docking energy was selected for further analysis. 


\section{Results}

\subsection{Stability of EGCG and GCG under DXR assay conditions}

EGCG and GCG are unstable, especially under neutral and basic conditions [19]. Because the DXR assay is performed usually in Tris- $\mathrm{HCl}$ buffer $(\mathrm{pH} 7.0)$ at $37^{\circ} \mathrm{C}$, we evaluated the stability of the two compounds under such conditions. The results (Fig. S1 in Supplementary Material) show that EGCG and GCG are indeed unstable in the Tris-HCl buffer at almost neutral pH: EGCG completely disappears after $2 \mathrm{~h}$ incubation at $37^{\circ} \mathrm{C}$ and GCG whose chemical stability is better than that of EGCG also decomposes quickly and only about $20 \%$ of it remains after $2 \mathrm{~h}$.

Fortunately, in the Tris- $\mathrm{HCl}$ buffer containing $1 \mathrm{mM} \mathrm{VC}$, the two compounds could be stable for at least $3 \mathrm{~h}$ at $37^{\circ} \mathrm{C}$. Therefore, the DXR assays testing the two compounds should be carried out in the presence of $1 \mathrm{mM} \mathrm{VC}$.

\subsection{Reliability of the pre-column derivatization HPLC method}

We have set up a pre-column derivatization HPLC method using DNPH as a derivative reagent to determine the DXR inhibitory activity of the theaflavins $[15,20]$. In this study we would extend it to measure the DXR inhibition of EGCG and GCG. The principle and the procedure of the method were given in the Fig. S2 in SM. We evaluated the reliability of the method in this study. The results show that the existence of EGCG/GCG would not interfere with the quantification of DXP (the HPLC profiles and retention times see Fig. S3 in SM).

\subsection{Measurement of CIAP inhibitory effects of the different compounds}


In the measurement of the inhibition of EGCG and GCG against DXR, we must use CIAP to hydrolyze DXP before DNPH derivatization. Therefore, whether EGCG/GCG and the other compounds like VC, baicalein, and Triton X-100 have any suppressive effects against this protein should be tested. To our delight, the results indicated that all the components have no inhibition against CIAP at all tested concentrations (see Fig. S4 in SM).

\subsection{DXR inhibition of EGCG/GCG with 2\% DMSO as a co-solvent}

With the verified HPLC method, we also tested if VC could have any influence on DXR activity. The results point out that VC exhibits no effect at all on the activity of DXR at $1 \mathrm{mM}$. Then, the DXR inhibitory activity of EGCG/GCG at a final concentration of $100 \mu \mathrm{M}$ was determined using $2 \%$ DMSO as a co-solvent and $1 \mathrm{mM} \mathrm{VC}$ as a protective agent. As expected, fosmidomycin that was used as the positive control shows $100 \%$ inhibition on DXR activity at $1.0 \mu \mathrm{M}$. The results also show that EGCG exhibits around 20\% inhibition against DXR, whereas GCG totally inhibits the activity of the target. Based on the experiments, we further determined the DXR inhibitory effects of the two compounds at different concentrations. The results, as given in Fig. 3 reveal that the two compounds display clear concentration dependent inhibitory manners against DXR. The $\mathrm{IC}_{50}$ values of them could therefore be calculated (Table 2), respectively. We could also find from the figure that EGCG possesses a weak inhibitory activity against DXR, with complete inhibition being at about $610 \mu \mathrm{M}$, while GCG shows a potent inhibitory activity and it could totally inhibit DXR at about $60 \mu \mathrm{M}$. We also measured the DXR suppressive activity of the two catechin compounds in the absence of the protective agent VC and the results depicted in Fig. 3 
manifest that the absence of VC in the assay mixture results in decrease of DXR inhibitory activity of both EGCG and GCG. This could be because that the presence of VC could protect

EGCG/GCG from oxidative decomposition. Same phenomena have been reported earlier [21,22].
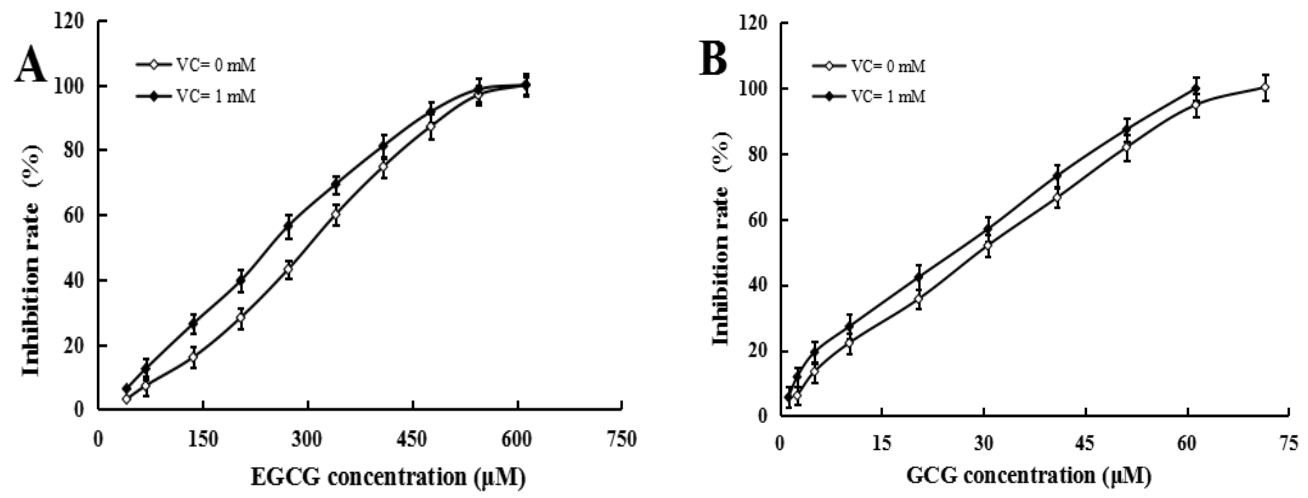

Fig. 3 The concentration dependent inhibitory manners of EGCG and GCG against DXR in the absence and presence of $1 \mathrm{mM}$ VC. A. EGCG; B. GCG.

Table $2 \mathrm{IC}_{50}$ values and inhibitory constants of EGCG/GCG against DXR.

\begin{tabular}{ccccccc}
\hline & \multicolumn{2}{c}{$\mathrm{IC}_{50}(\mu \mathrm{M})$} & \multicolumn{2}{c}{ NADPH } & \multicolumn{2}{c}{ DXP } \\
\cline { 2 - 6 } & $\mathrm{A}^{\mathrm{a}}$ & $\mathrm{B}$ & $K \mathrm{i}(\mu \mathrm{M})$ & Kinetics & $K \mathrm{i}(\mu \mathrm{M})$ & Kinetics \\
\hline EGCG & $281.5 \pm 4.9$ & $209.8 \pm 4.1$ & $179.4 \pm 5.3$ & & $148.3 \pm 5.5$ & \\
GCG & $36.5 \pm 3.6$ & $27.5 \pm 3.8$ & $23.4 \pm 4.4$ & & Competitive \\
Fosmidomycin & & & & & \\
\end{tabular}

${ }^{\mathrm{a}}$ The data obtained in the absence of VC; ${ }^{\mathrm{b}}$ Fosmidomycin was used as a positive control $[11,13]$.

\subsection{DXR inhibitory activity of EGCG/GCG with $0.01 \%$ Triton X-100 as a surfactant}


Some flavonoid compounds such as myricetin, baicalein, and quercetin et al. have recently been reported to restrain DXR activity with quite high activity $\left(\mathrm{IC}_{50}<5 \mu \mathrm{M}\right)$ when using DMSO as a co-solvent. However, their inhibitory effects against DXR substantially lowered when the non-ionic detergent Triton X-100 was employed to enhance the solubility of the flavonoids. Thus these compounds were considered to be non-specific inhibitors of DXR [23]. In fact, Triton $\mathrm{X}-100$ is often utilised as a dispersing agent to distinguish between specific and promiscuous inhibition [24-28]. We therefore assayed the DXR inhibitory activity of EGCG and GCG using $0.01 \%$ Triton $\mathrm{X}-100$ as a surfactant to elucidate if they were aggregating inhibitors of DXR. We first tested the influence of Triton X-100 on DXR and found that the detergent had no effect on the activity of DXR at a concentration of $0.1 \%$. Then two different concentrations for each of the two compounds were set to evaluate its inhibition against $\mathrm{DXR}$, one was close to its $\mathrm{IC}_{50}$ value and the other was about twice of it. From the data given in Table 3 we could see that for each of the compound, no noticeable difference of DXR inhibition could be observed in presence and absence of the detergent at both concentrations. We also selected baicalein as a positive control since it has been demonstrated to be a non-specific inhibitor of DXR [23] and had no effect on the activity of CIAP at concentrations up to $500 \mu \mathrm{M}$ (Data see Fig. S4 in SM). The results (Table 3) showed that the presence of Triton X-100 could significantly reduce its inhibition potency. Therefore, according to the criteria set up by Shoichet and co-workers [27], the above observation suggested that EGCG and GCG could inhibit DXR via a specific manner. 
Table 3 Influence of $0.01 \%$ Triton X-100 on inhibition of E. coli DXR with EGCG and GCG.

\begin{tabular}{|c|c|c|c|c|c|c|}
\hline & \multirow{2}{*}{ Conc $(\mu \mathrm{M})$} & \multicolumn{2}{|c|}{ Inhibition (\%) } & \multirow{2}{*}{ Conc $(\mu \mathrm{M})$} & \multicolumn{2}{|c|}{ Inhibition (\%) } \\
\hline & & No Triton ${ }^{a}$ & Triton & & No Triton ${ }^{\mathrm{a}}$ & Triton \\
\hline EGCG & 200 & $38.7 \pm 4.6$ & $38.2 \pm 3.1$ & 400 & $80.4 \pm 3.8$ & $79.5 \pm 4.5$ \\
\hline GCG & 20 & $40.3 \pm 3.4$ & $41.7 \pm 3.9$ & 40 & $70.6 \pm 4.1$ & $72.1 \pm 3.4$ \\
\hline Baicalein $^{\mathrm{b}}$ & 50 & $49.8 \pm 4.2$ & $5.8 \pm 4.7$ & 100 & $76.9 \pm 4.9$ & $8.2 \pm 4.1$ \\
\hline
\end{tabular}

${ }^{\mathrm{a}}$ Using $2 \%$ DMSO as a co-solvent. ${ }^{\mathrm{b}}$ Baicalein was used as a positive control [23].

\subsection{Analysis of the particle size by DLS}

DLS is a widely applied technique in material sciences to determine particle sizes in solutions [27,29]. Generally, DLS measurements generate two parameters, one is the scattering intensity which represents particle concentration; the other is average particle size calculated from the autocorrelation functions of the scattered laser light. In this study, we took this method to detect if there were any particles formed in the samples in which EGCG or GCG was diluted to an end concentration of around 2-3 times of its $\mathrm{IC}_{50}$ value in $50 \mathrm{mM}$ Tris- $\mathrm{HCl}$ buffer ( $\left.\mathrm{pH} 7.0\right)$ in absence or presence of DXR (4 $\mu \mathrm{g} / \mathrm{mL})$. Baicalein was also selected as a positive control since it is a non-specific inhibitor of DXR [23]. From the data shown in Table 4, we could find that no obvious particle was detectable in EGCG/GCG samples, while in the sample containing baicalein, the aggregation of DXR was able to be clearly observed. 
Table 4 Dynamic Light Scattering of EGCG/GCG plus DXR in $50 \mathrm{mM}$ Tris-HCl buffer (pH 7.0) containing $1 \mathrm{mM} \mathrm{VC}$

\begin{tabular}{|c|c|c|c|c|c|}
\hline & \multirow{4}{*}{ Conc $(\mu \mathrm{M})^{\mathrm{a}}$} & \multicolumn{2}{|c|}{ Without DXR } & \multicolumn{2}{|c|}{ With $4 \mu \mathrm{g} / \mathrm{mL}$ DXR } \\
\hline & & Count Rate & \multicolumn{3}{|c|}{ Count Rate } \\
\hline & & & Size $(\mathrm{nm})$ & & Size $(n m)$ \\
\hline & & $(\mathrm{KHz})$ & & $(\mathrm{KHz})$ & \\
\hline EGCG & 500 & $3.2 \pm 0.4$ & $\mathrm{~N} / \mathrm{A}^{\mathrm{d}}$ & $5.4 \pm 0.5$ & N/A \\
\hline GCG & 80 & $2.4 \pm 0.5$ & N/A & $4.2 \pm 0.4$ & N/A \\
\hline Baicalein $^{\mathrm{b}}$ & 200 & $2.6 \pm 0.5$ & N/A & $296 \pm 19.7$ & $429.2 \pm 84.7$ \\
\hline Blank $^{\mathrm{c}}$ & --- & $2.4 \pm 0.5$ & N/A & $4.8 \pm 0.4$ & N/A \\
\hline
\end{tabular}

${ }^{\mathrm{a}}$ Final concentration of DMSO was $2 \%(\mathrm{v} / \mathrm{v}) ;{ }^{\mathrm{b}}$ Baicalein was used as a positive control [23];

${ }^{\mathrm{c}}$ Blank: 2\% DMSO plus $1 \mathrm{mM} \mathrm{VC;}{ }^{\mathrm{d}} \mathrm{N} / \mathrm{A}$ : not available.

\subsection{Interaction of EGCG/GCG with DXR monitored by UV spectrometry}

EGCG has been proven to inhibit $\beta$-ketoacyl-acyl carrier protein reductase (FabG)

non-specifically because it was able to induce aggregation of the protein, leading to increase of the turbidity of an FabG solution (around 2-7 $\mu \mathrm{M}$ protein in phosphate buffer, $\mathrm{pH}$ 7.0) containing $200 \mu \mathrm{M}$ EGCG [18]. In order to get more data to support EGCG and GCG can inhibit DXR in a specific mechanism, we also monitored the $\mathrm{A}_{400}$ and the DLS of DXR solutions (around $3 \mu \mathrm{M}$ in $2.5 \mathrm{mM}$ phosphate buffer, $\mathrm{pH}$ 7.0) containing $200 \mu \mathrm{M}$ EGCG or GCG in the absence and presence of $1 \mathrm{mM} \mathrm{VC}$ at room temperature. The results show that for the solutions containing no VC, their $\mathrm{A}_{400}$ went up slowly (Fig. 4) and their appearances turned gradually from colorlessness 
to pale yellow, and finally to yellow; and for the solutions with $\mathrm{VC}$, both of their $\mathrm{A}_{400}$ and appearances kept unchanged as time elapsed. Meanwhile, DLS determination of these solutions manifests that no detectable particles formed during the process. We also measured the $\mathrm{A}_{400}$ of EGCG/GCG solutions ( $200 \mu \mathrm{M}$ in $2.5 \mathrm{mM}$ phosphate buffer, $\mathrm{pH} 7.0)$ in the absence and presence of $1 \mathrm{mM} \mathrm{VC}$ and observed the same phenomenon as those of DXR solutions: when there was no VC in the solutions, their $\mathrm{A}_{400}$ arose gradually and color changed slowly; whereas neither $\mathrm{A}_{400}$ increase nor appearance change was able to be followed when $1 \mathrm{mM} \mathrm{VC}$ was present in the solutions. Based on these data, we would say that the increase of $\mathrm{A}_{400}$ and the color change of the DXR-EGCG/GCG solutions without VC result from the oxidative decomposition of EGCG/GCG and not due to the promiscuous interaction between DXR and the catechins. These observations are in agreement with the results in the Triton X-100 experiment and the DLS analysis that EGCG and GCG inhibit DXR in a specific mode.

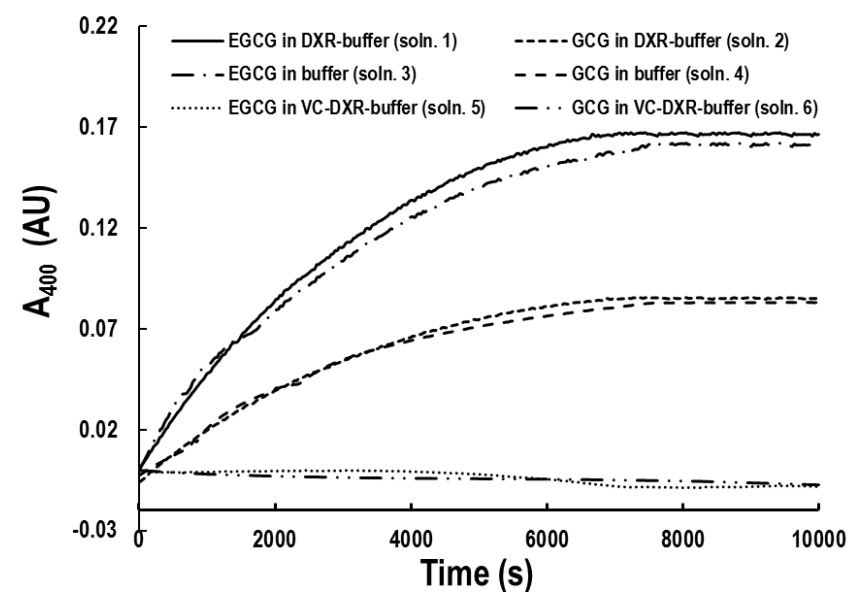

Fig. 4 The $\mathrm{A}_{400}$-Time curves of $2.5 \mathrm{mM}$ phosphate buffer solutions ( $\mathrm{pH}$ 7.0) containing different additives. The concentrations of EGCG and GCG were fixed at $200 \mu \mathrm{M}, \mathrm{DXR}$ and VC were 3 $\mu \mathrm{M}$ and $1 \mathrm{mM}$, respectively. All the curves were recorded at $37^{\circ} \mathrm{C}$. Because the curves of the 
solns. 5-10 almost totally overlapped with each other, only curves of solns. 5 and 6 were remained to simplify the plot. The curves of the solns. 5 to 10 see Fig. S5 in SM.

\subsection{Determination of the inhibition kinetics of EGCG and GCG against DXR}

To elucidate the inhibitory mechanism of EGCG and GCG against DXR, the initial enzyme kinetics was studied over fixed EGCG/GCG concentrations and at different DXP/NADPH concentrations through the evaluated HPLC method. Lineweaver-Burk (LB) graphics were produced by plotting the reciprocal of the reaction velocity against the reciprocal of the concentrations of DXP/NADPH. The results depicted in Figs. 5A and 5B demonstrate that both EGCG and GCG are competitive inhibitors of DXR when DXP is the altered substrate and give uncompetitive patterns against NADPH. The $K_{\mathrm{i}}$ values of the two compounds against NADPH and DXP respectively were calculated accordingly and listed in Table 2.
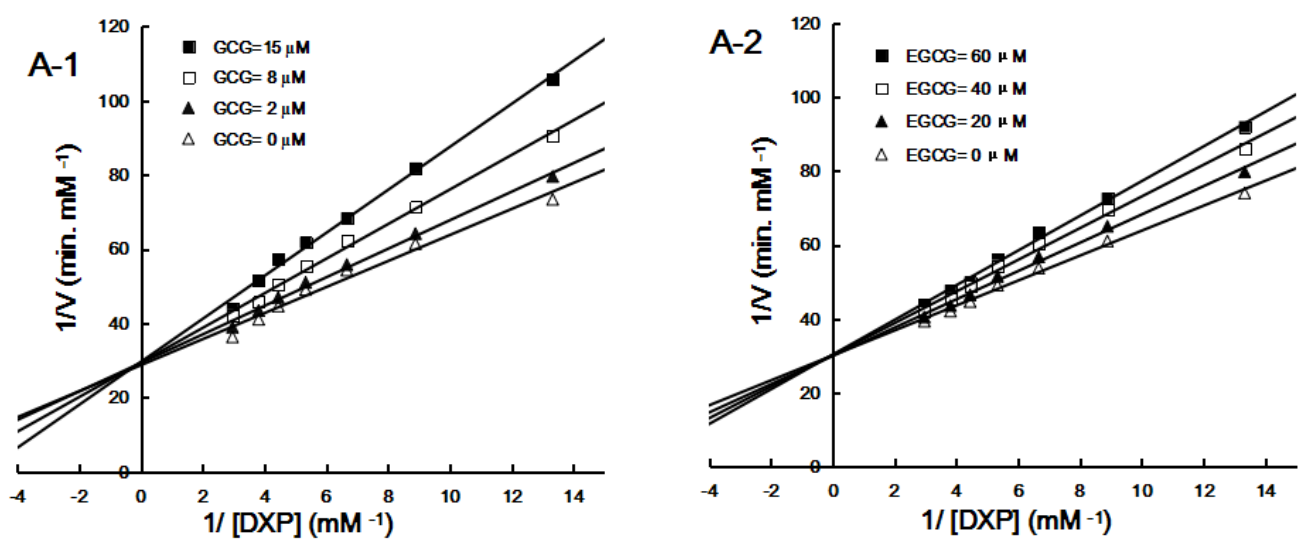

Fig. 5A Lineweaver-Burk plots of DXR against DXP in the absence and presence of GCG (A-1) and EGCG (A-2). Assay mixtures containing $50 \mathrm{mM}$ Tris- $\mathrm{HCl}$ (pH 7.0), $5 \mathrm{mM} \mathrm{MgCl} 2,1 \mathrm{mM} \mathrm{VC}$, the catechins (GCG: 0, 2, 8, $15 \mu \mathrm{M}$; EGCG: 0, 20, 40, or $60 \mu \mathrm{M}$ ), $0.5 \mathrm{mM}$ NADPH, $1.5 \mu \mathrm{g} / \mathrm{mL}$ DXR, and 0.075 to $0.3375 \mathrm{mM} \mathrm{DXP}$ in a final volume of $100 \mu \mathrm{L}$ were incubated for $10 \mathrm{~min}$ at 
$37^{\circ} \mathrm{C}$ before the mixtures were hydrolyzed with CIAP, derivatized with DNPH, and analyzed using the HPLC method.
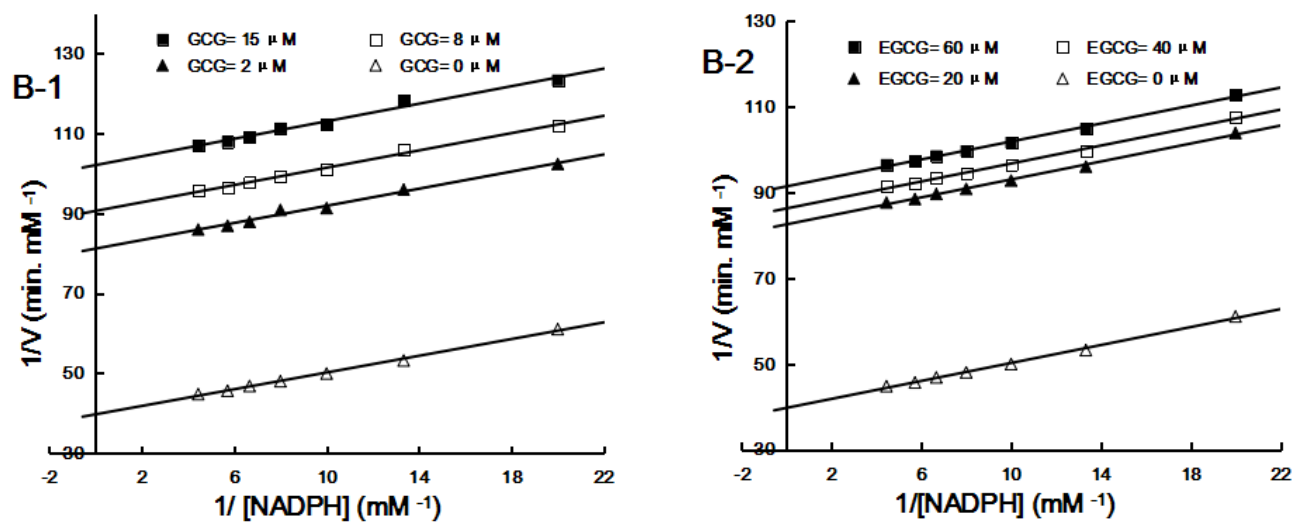

Fig. 5B Lineweaver-Burk plots of DXR against NADPH in the absence and presence of GCG (B-1) and EGCG (B-2). Assay mixtures containing $50 \mathrm{mM}$ Tris- $\mathrm{HCl}(\mathrm{pH} 7.0), 5 \mathrm{mM} \mathrm{MgCl} 2,1$ mM VC, the catechins (GCG: 0, 2, 8, $15 \mu \mathrm{M}$; EGCG: 0, 20, 40, or $60 \mu \mathrm{M}$ ), $1 \mathrm{mM}$ DXP, 1.5 $\mu \mathrm{g} / \mathrm{mL}$ DXR, and 0.05 to $0.275 \mathrm{mM}$ NADPH in a final volume of $100 \mu \mathrm{L}$ were incubated for 10 min at $37^{\circ} \mathrm{C}$ before the mixtures were hydrolyzed with CIAP, derivatised with DNPH, and analyzed using the HPLC method.

\section{Docking result of EGCG and GCG to DXR}

In order to get more information about the interaction between the compounds and DXR so that we can reasonably explain the DXR inhibition of them, we carried out some docking experiments to mimic the binding manners of EGCG and GCG to DXR. The results prove that both the compounds locate at the active hydrophobic pocket of DXR, as shown in Fig. S6 in SM. The difference is that GCG is able to adopt a favorable conformation, allowing the deep insertion of the intact molecule into the active cavity with its pyrogalloyl moiety directing to $\mathrm{Mg}^{2+}$ present in 
the active site, whereas EGCG cannot adopt this kind of conformation, leading to the result that the main body of the molecule can only stay at the entrance of the cavity with its pyrogalloyl moiety orienting to the distal metal ion. The close-up views of the docking results depicted in Fig. S7 in SM display the interactions between the inhibitors and the cofactors/the residues. The global impression we could get from the figure is that there are much more wire balls in A than in $\mathrm{B}$, indicating that GCG generates much stronger interactions with the target protein and the cofactors than EGCG does. In particular, there is a green wire ball at the up right corner of Fig. S7A, but no in Fig. S7B, suggesting that only GCG could produce close contact with $\mathrm{Mg}^{2+}$, probably through chelating its pyrogalloyl moiety with the metal ion. Further analyses of the figure result in the following observations: i) besides its coordination with the metal ion, GCG could bind to the enzyme through eight residues: Trp211, Ser212, Met213, Gly214, Ile217, Asp149, Glu151, and Pro273. It forms two H-bonds (purple bead wires in the figure) with DXR and produces close contact with the other six residues (yellow wire balls). One of the H-bonds is between its 7-OH and Pro273, the other is between the carbonyl of the gallate group and Gly214. In addition, GCG also formed an H-bond and quite much close contact with NADPH (blue wire balls); ii) EGCG could bind to DXR at seven residues: Val101, Lys124, Met213, Gly214, Arg215, Lys216, and Ile217. It totally forms four H-bonds, three with DXR residues and one with the cofactor NADPH and generates close contact with the other five residues. Among the three $\mathrm{H}$-bonds with the enzyme, one is between Lys124 and its $\mathrm{OH}$ of the pyrogalloyl moiety, and the other two are separately between Gly214 and Lys216 and the two OHs of the gallate moiety. The forth one forms between the pyrophosphate moiety of NADPH and its 5-OH; iii) Five of the eight 
GCG binding sites, namely Trp211, Met213, Ile217, Asp149, and Glu151 are also the binding sites for DXP. These residues, especially Trp211 and Met213 are key to the activity of DXR [30-33]. For EGCG, only two of its seven binding residues, Lys124, Met213 are DXP binding sites. Furthermore, the energy parameters and the $K \mathrm{i}$ values obtained from the docking experiments (Table $\mathrm{S} 1$ in the SM) showed that GCG could bind much more tightly to the active sites of DXR than EGCG because GCG was able to produce stronger interaction with DXR than EGCG. All these data could reasonably explain that GCG shows much more potent inhibition against DXR than EGCG does.

\section{Discussion}

DXR has been believed to be an attractive target for seeking novel antimicrobial agents, and up to now, fosmidomycin $\mathbf{5}$ and its congener FR900098 $\mathbf{6}$ have been determined as the most potent inhibitors of this protein $[9,11]$. These two compounds, however, show problems in their druggability such as short plasma half-life (shorter than $1 \mathrm{~h}$ ), poor bioavailability, and metabolic liability, which have obstructed their in vivo application as DXR inhibitors [34]. Therefore, extensive investigations have been carried out to acquire new inhibitors of this enzyme that should possess better druggable properties than $\mathbf{5}$ and $\mathbf{6}$.

Many plants have been utilised either to prevent the spread of the infectious diseases or to treat various infections in different countries for centuries. However, this area has remained largely unexplored in screening DXR inhibitors. Numerous reports on the bioactivities of the catechins with special emphasis on their antimicrobial effects have been published [35,36], but the 
antimicrobial mode of action of the catechins, in particularly the major active compounds EGCG and GCG, is still obscure. Thus we decided to determine whether EGCG and GCG would suppress the activity of DXR and try to further elucidate the antimicrobial mechanism of them.

Generally, the most convenient way to monitor the DXR reaction is photometric method because this reaction involves the dehydrogenation of stoichiometric amounts of NADPH that has absorption at $340 \mathrm{~nm}$. But this protocol is not applicable in our study since the use of EGCG or GCG could interfere with the determination. We therefore adopted the HPLC method previously established by us $[15,20]$ and validated its reliability. In addition, we found a way to stabilize EGCG and GCG under DXR assay conditions. Based on these results, we measured the inhibition of the two compounds against DXR and the data indicate that EGCG exhibits lower DXR inhibitory activity with an $\mathrm{IC}_{50}$ being around $200 \mu \mathrm{M}$, whereas GCG shows strong inhibition against the target with an $\mathrm{IC}_{50}$ value of $27.5 \mu \mathrm{M}$. Thus, the DXR inhibitory activities of EGCG and GCG apparently correspond to their configuration at C-2, with $2 S$ (GCG) being much better than $2 R$ (EGCG). Same phenomenon has been observed on the suppressive capacity of the two compounds against Bacillus cereus [8]. Furthermore, the two compounds restrict the activity of DXR in clear dose-dependent manners (Fig. 3).

There have been a number of research articles on the bioactivities of polyphenols, but the non-specific restriction of this kind of compounds against various proteins has also been reported recently [24-29]. Non-specific inhibition of DXR by some flavonoids and by synthetic compounds holding a catechol moiety has also been determined [23,27]. The reason of this is now attributed to the interaction between flavonoids and proteins to generate aggregates of 
enzymes, resulting in reduction or even totally loss of enzymatic activities [26,27]. Shoichet and co-workers have set up some criteria to distinguish whether a compound is a promiscuous or aggregation-based inhibitor [27].

EGCG and GCG both contain a flavanol moiety in the scaffold, thus they could also produce non-specific inhibition against DXR. In view of this situation, we performed extra assays to decide whether the inhibition of the two compounds is specific according to Shoichet's criteria [27]. Because the Triton X-100 experiment showed that the DXR inhibition of EGCG and GCG is not sensitive at all to the detergent and the results of DLS analysis manifest no aggregate of the enzyme could be detected in the assay mixtures, we would conclude that the tested compounds produce specific inhibition on DXR. Furthermore, our data also show that EGCG and GCG do not have any inhibitory ability against CIAP, an enzyme totally unrelated with DXR, which could be supportive for the specific inhibition of two compounds against DXR.

It has been reported that EGCG is a potent inhibitor of the bacterial type II fatty-acid synthase, especially FabG and trans-2-enoyl-acyl carrier protein reductase [7]. Li et al. studied the interaction of EGCG with FabG and found that EGCG exhibited promiscuous inhibition against FabG because it was able to induce aggregation of FabG [18]. To further verify EGCG and GCG can produce specific DXR inhibition, we determined the variation of $\mathrm{A}_{400}$ of a DXR solution containing EGCG or GCG employing the exact method established by Li [18]. We indeed observed increase of $\mathrm{A}_{400}$ and change in color of the solution without $\mathrm{VC}$, but the phenomena were able to be completely avoided by adding VC, an antioxidant to the solution. Thus, these alterations should be due to the oxidative decomposition of the catechins, not owing to the 
aggregation of the enzyme. DLS analysis also gave negative results. These data provide further evidence that EGCG and GCG are specific inhibitors of DXR.

Having characterized that EGCG and GCG exactly produce specific restriction on DXR, we determined their inhibition kinetics using Lineweaver-Burk double-reciprocal plots (Figs. 5A and 5B). The results display that they are both competitive inhibitors of DXR with respect to DXP and un-competitive inhibitors with respect to NADPH. It is quite surprising that EGCG and GCG are competitive inhibitors of DXR versus DXP because the sole structural similarity between EGCG/GCG and DXP is that they share the vicinal diol substructure. It might be the structural feature that makes EGCG/GCG the competitive inhibitors of DXR versus DXP since it has been suggested that the vicinal diol substructure could be the key for DXP to coordinate with $\mathrm{Mg}^{2+}$ bound to DXR [16,37,38], and that the catechol group might be important for DXR inhibitors to chelate with $\mathrm{Mg}^{2+}$ present in the active site of the protein [28]. The docking experiments strongly support that GCG and EGCG are competitive inhibitors of DXR versus DXP because they could bind at the binding sites of DXP. Moreover, GCG could produce interaction with $\mathrm{Mg}^{2+}$ bound to DXR as well. The un-competitive inhibition mode of EGCG and GCG against NADPH was probably reasonable because 1) it has been demonstrated that E. coli DXR catalyzed the conversion reaction through an ordered Ping Pong mechanism, with NADPH binding first [39]; 2) the docking data show either GCG or EGCG could form H-bond and also produce close contact with NADPH.

\section{Conclusion}


In summary, the data obtained in this study show that the compound GCG possesses potent, specific inhibition against DXR; its epimer, EGCG also shows same, but relatively weak effect. This research discloses the antibacterial mechanism of the two catechin compounds and may provide a new type of DXR inhibitor that is totally different from fosmidomycin.

\section{Conflict of interest}

There are no conflicts of interest to declare.

\section{Acknowledgments}

This work was supported by the National Science Foundation of China (NSFC, 21172179, 21402152), the Program for Changjiang Scholars and Innovative Research Team in University

(No. IRT_15R55), and the Graduate Innovation and Creativity Funds of Northwest University (YZZ15060). We gratefully acknowledge Prof. Yong-Kuan Gong and Dr. Shi-Ping Zhang of School of Chemistry and Materials Science, Northwest University for their kind help in particle size analysis.

\section{References}

1. J. Peterson, J. Dwyer, S. Bhagwat, D. Haytowitz, J. Holden, A.L. Eldridge, J. Aladesanmi, Major flavonoids in dry tea, J. Food Compos. Anal. 18 (2005) 487-501. 
2. Y. Cui, Y.J. Oh, J. Lim, M. Youn, I. Lee, H.K. Pak, S. Park, AFM study of the differential inhibitory effects of the green tea polyphenol (-)-epigallocatechin-3-gallate (EGCG) against Gram-positive and Gram-negative bacteria, Food Microbiol. 29 (2012) 80-87.

3. M. Daglia, Polyphenols as antimicrobial agents, Curr. Opin. Biotech. 23 (2012) 174-181.

4. T. Shimamura, W.H. Zhao, Z.Q. Hu, Mechanism of action and potential for use of tea catechin as an antiinfective agent, Antiinfect Agents Med Chem. 6 (2007) 57-62.

5. H. Arakawa, M. Maeda, S. Okubo, T. Shimamura, Role of hydrogen peroxide in bactericidal action of catechin, Biolog. Pharm. Bul. 27 (2004) 277-281.

6. Y. Sugita-Konishi, Y. Hara-Kudo, F. Amano, T. Okubo, N. Aoi, M. Iwaki, S. Kumagai, Epigallocatechin gallate and gallocatechin gallate in green tea catechins inhibit extracellular release of Vero toxin from enterohemorrhagic Escherichia coli O157: H7, BBA-Gen. Subjects 1472 (1999) 42-50.

7. Y. M. Zhang, C.O. Rock, Evaluation of epigallocatechin gallate and related plant polyphenols as inhibitors of the FabG and FabI reductases of bacterial type II fatty-acid synthase, Biol. Chem. 279 (2004) 30994-31001.

8. M. Friedman, P.R. Henika, C.E. Levin, R.E. Mandrell, N. Kozukue, Antimicrobial activities of tea catechins and theaflavins and tea extracts against Bacillus cereus, J. Food Protect. 69 (2006) 354-361.

9. J. Pérez-Gil, M. Rodríguez-Concepción, Metabolic plasticity for isoprenoid biosynthesis in bacteria, Biochem. J. 452 (2013) 19-25. 
10. L. Zhao, W.C. Chang, Y. Xiao, H.W. Liu, P. Liu, Methylerythritol phosphate pathway of isoprenoid biosynthesis, Annu. Rev. Biochem. 82 (2013) 497-530.

11. H. Jomaa, J. Wiesner, S. Sanderbrand, B. Altincicek, C. Weidemeyer, M. Hintz, D. Soldati, Inhibitors of the nonmevalonate pathway of isoprenoid biosynthesis as antimalarial drugs, Science 285 (1999) 1573-1576.

12. M.A. Missinou, S. Borrmann, A. Schindler, S. Issifou, A.A. Adegnika, P.B. Matsiegui, H. Jomaa, Fosmidomycin for malaria, Lancet 360 (2002) 1941-1942.

13. J. Kaiser, M. Yassin, S. Prakash, N. Safi, M. Agami, S. Lauw, J. Safi, Anti-malarial drug targets: screening for inhibitors of 2C-methyl-D-erythritol 4-phosphate synthase (IspC protein) in Mediterranean plants, Phytomedicine 14 (2007) 242-249.

14. X. Hui, H. Liu, F.L. Tian, F.F. Li, H. Li, W.Y. Gao, Inhibition of green tea and the catechins against 1-deoxy-D-xylulose 5-phosphate reductoisomerase, the key enzyme of the mep terpenoid biosynthetic pathway, Fitoterapia 113 (2016) 80-84.

15. X. Hui, Q. Yue, D.D. Zhang, H. Li, S.Q. Yang, W.Y. Gao, Antimicrobial mechanism of theaflavins: They target 1-deoxy-D-xylulose 5-phosphate reductoisomerase, the key enzyme of the MEP terpenoid biosynthetic pathway. Sci. Rep. 2016, 6, 38945.

16. H. Li, J. Tian, H. Wang, S.Q. Yang, W.Y. Gao, An Improved Preparation of D-Glyceraldehyde 3-Phosphate and Its Use in the Synthesis of 1-Deoxy-D-xylulose 5-Phosphate, Helv. Chim. Acta. 93 (2010) 1745-1750. 
17. H. Li, J. Tian, W. Sun, W. Qin, W.Y. Gao, Mechanistic insights into 1-deoxy-D-xylulose 5-phosphate reductoisomerase, a key enzyme of the MEP terpenoid biosynthetic pathway, FEBS. J. 280 (2013) 5896-5905.

18. B.H. Li, R. Zhang, Y.T. Du, Y.H. Sun, W.X. Tian, Inactivation mechanism of the $\beta$-ketoacyl-[acyl carrier protein] reductase of bacterial type-II fatty acid synthase by epigallocatechin gallate, Biochem. Cell Biol. 84 (2006) 755-762.

19. Y.L. Su, L.K. Leung, Y.Huang, Z.Y. Chen, Stability of tea theaflavins and catechins, Food Chem. 83 (2003) 189-195.

20. Y. Hu, X.J. Wang, H. Li and W.Y. Gao, Determination of steady-state kinetic parameters of 1-Deoxy-D-xylulose-5-phosphate synthase by pre-column derivatization high performance liquid chromatography using 2,4-dinitrophenylhydrazine as a derivative reagent. Chin. J. Anal. Chem., 40 (2012) 1859-1864. (English version)

21. T. Hatano, M. Tsugawa, M. Kusuda, S. Taniguchi, T. Yoshida, S. Shiota, T. Tsuchiya, Enhancement of antibacterial effects of epigallocatechin gallate, using ascorbic acid, Phytochemistry 69 (2008) 3111-3116.

22. Y. Gao, W. Li, L.Y. Jia, B. Li, Y.C. Chen, Y. Tu, Enhancement of (-)-epigallocatechin3-gallate and theaflavin-3-3'-digallate induced apoptosis by ascorbic acid in human lung adenocarcinoma SPC-A-1 cells and esophageal carcinoma Eca-109 cells via MAPK pathways, Biochem. Bioph. Res. Co. 438 (2013) 370-374. 
23. D. Tritsch, C. Zinglé, M. Rohmer, C. Grosdemange-Billiard, Flavonoids: True or promiscuous inhibitors of enzyme? The case of deoxyxylulose phosphate reductoisomerase, Bioorg. Chem. 59 (2015) 140-144.

24. B.Y. Feng, A. Simeonov, A. Jadhav, K. Babaoglu, J. Inglese, B.K. Shoichet, C.P. Austin, A high-throughput screen for aggregation-based inhibition in a large compound library, J. Med. Chem. 50 (2007) 2385-2390.

25. B.Y. Feng, B.K. Shoichet, A detergent-based assay for the detection of promiscuous inhibitors, Nat. Protoc. 1 (2006) 550-553.

26. S.L. McGovern, B.T. Helfand, B. Feng, B.K. Shoichet, A specific mechanism of nonspecific inhibition, J. Med. Chem. 46 (2003) 4265-4272.

27. J. Seidler, S.L. McGovern, T.N. Doman, B.K. Shoichet, Identification and prediction of promiscuous aggregating inhibitors among known drugs, J. Med. Chem. 46 (2003) $4477-4486$.

28. C. Zinglé, D. Tritsch, C. Grosdemange-Billiard, M. Rohmer, Catechol-rhodanine derivatives: Specific and promiscuous inhibitors of Escherichia coli deoxyxylulose phosphate reductoisomerase (DXR), Bioorg. Med. Chem. 22 (2014) 3713-3719.

29. L. Pohjala, P. Tammela, Aggregating behavior of phenolic compounds - a source of false bioassay results? Molecules 17 (2012) 10774-10790.

30. L. Deng, J. Diao, P. Chen, V. Pujari, Y. Yao, G. Cheng, Y. Song, Inhibition of 1-deoxy-D-xylulose-5-phosphate reductoisomerase by lipophilic phosphonates: SAR, QSAR, and crystallographic studies, J. Med. Chem. 54 (2011) 4721-4734. 
31. A. Mac Sweeney, R. Lange, R.P. Fernandes, H. Schulz, G.E. Dale, A. Douangamath, C. Oefner, The crystal structure of E. coli 1-deoxy-D-xylulose-5-phosphate reductoisomerase in a ternary complex with the antimalarial compound fosmidomycin and NADPH reveals a tight-binding closed enzyme conformation, J. Mol. Biol. 345 (2005) 115-127.

32. S. Yajima, K. Hara, J.M. Sanders, F. Yin, K.Ohsawa, J. Wiesner, E. Oldfield, Crystallographic structures of two bisphosphonate: 1-deoxyxylulose-5-phosphate reductoisomerase complexes, J. Am. Chem. Soc. 126 (2004) 10824-10825.

33. S. Yajima, T. Nonaka, T. Kuzuyama, H. Seto, K. Ohsawa, Crystal Structure of 1-Deoxy-D-xylulose 5-phosphate Reductoisomerase Complexed with Cofactors:-Implications of a Flexible Loop Movement upon Substrate Binding, J. Biochem. 131 (2002) 313-317.

34. M.C. Chang, J.D. Keasling, Production of isoprenoid pharmaceuticals by engineered microbes, Nat. Chem. Biol. 2 (2006) 674-681.

35. S. Quideau, D. Deffieux, C. Douat-Casassus, L. Pouysegu, Plant polyphenols: chemical properties, biological activities, and synthesis, Angew. Chem. Int. Ed. 50 (2011) 586-621.

36. M. Friedman, Overview of antibacterial, antitoxin, antiviral, and antifungal activities of tea flavonoids and teas, Mol. Nutr. Food Res. 51 (2007) 116-134.

37. J.F. Hoeffler, D. Tritsch, C. Grosdemange-Billiard, M. Rohmer, Isoprenoid biosynthesis via the methylerythritol phosphate pathway, Eur. J. Biochem. 269 (2002) 4446-4457. 
38. A. Wong, J.W. Munos, V. Devasthali, K.A. Johnson, H.W. Liu, Study of 1-deoxy-D-xylulose-5-phosphate reductoisomerase: synthesis and evaluation of fluorinated substrate analogues, Org. Lett. 6 (2004) 3625-3628.

39. A.T. Koppisch, D.T. Fox, B.S. Blagg, C.D. Poulter, E. coli MEP synthase: steady-state kinetic analysis and substrate binding, Biochemistry 41 (2002) 236-243. 


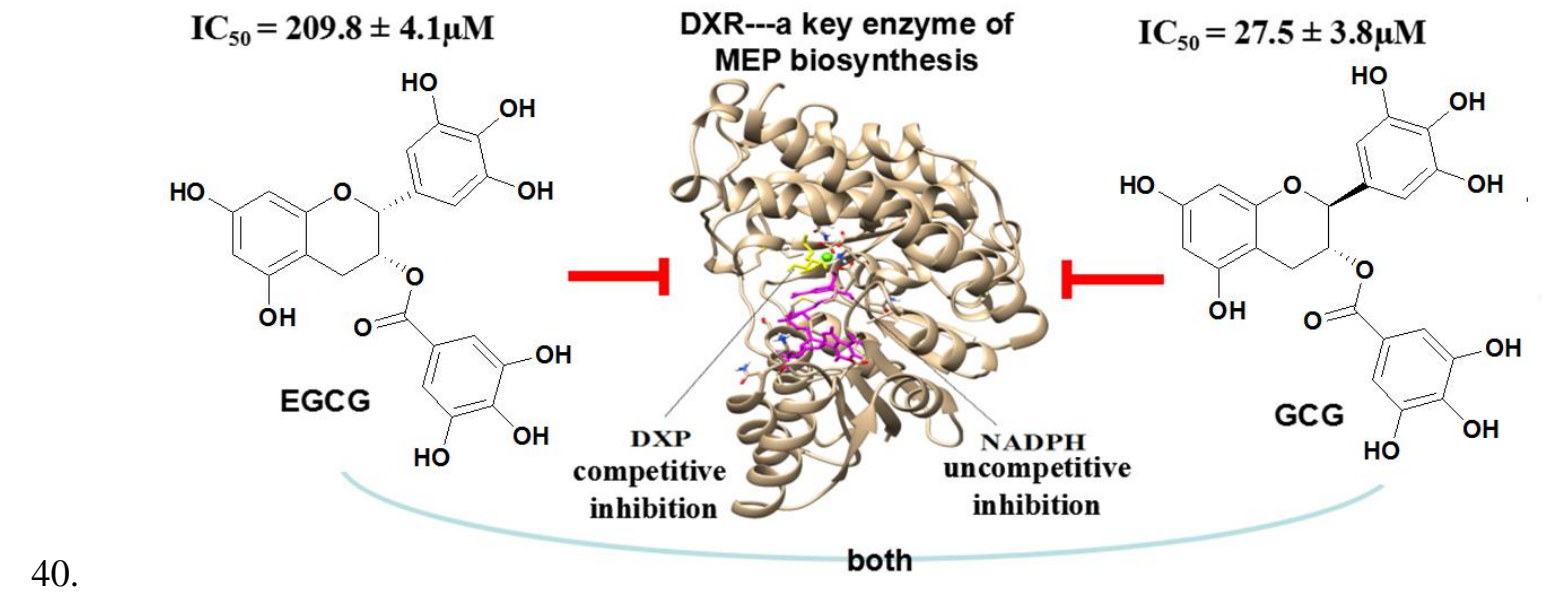

